Distinct Neural Responses to Social and Semantic Violations: An N400 Study

Natalie R. Weimer

Sheri L. Clark

Antonio L. Freitas

Stony Brook University

In press (as of December, 2018) at

International Journal of

Psychophysiology 


\begin{abstract}
We investigated event-related brain potential correlates of encountering context-incongruent social information. Building on evidence that information semantically incongruent with its context elicits an N400 response (a prominent negative-going deflection in the ongoing electroencephalogram; EEG), we hypothesized that statements incongruent (relative to congruent) with basic standards of amicable treatment by others (e.g., "Your friend breaks your computer and then laughs [apologizes]") would elicit larger-amplitude N400 responses. EEG was recorded from $\mathrm{N}=20$ undergraduates while they viewed 106 semantic-dimension and 106 social-dimension sentences. We obtained the classic N400 effect to semantic violations, but we did not observe greater N400 amplitudes to incongruent than to congruent social-dimension sentences. Our findings of N400 modulation by semantic violations but not social norm violations help clarify potential boundary conditions for eliciting the N400.
\end{abstract}

Keywords: N400, social violations, semantic violations, ERPs 


\section{Distinct Neural Responses to Social and Semantic Violations: An N400 Study}

Whether reading a book, talking with a friend, or watching the news, deriving meaning from information involves integrating multiple sources of knowledge. To interpret the sentence, "The earth is flat," for example, a person must access knowledge about the meaning of words and about the realities of the world (Hagoort, Hald, Bastiaansen, \& Petersson, 2004). Whereas the word "flat" does not present a linguistic violation, it conflicts with common knowledge that the earth is round. Thus, a phrase or sentence a person is interpreting may be regarded as false because it contains a semantic violation (based on knowledge of words) or a world-knowledge violation (based on knowledge of the world). What remains unclear is how these linguistic and non-linguistic sources of knowledge are accessed and integrated during online language comprehension.

One of the ways researchers have attempted to address the issue is with the N400. The N400 response is a negative-going deflection in the ongoing electroencephalogram (EEG) peaking approximately $400 \mathrm{~ms}$ after stimulus presentation. The amplitude of the N400 increases as the integration of information becomes increasingly difficult (e.g., Brown \& Hagoort, 1993; Hagoort et al., 2004; Van Berkum, Holleman, Nieuwland, Otten, \& Murre, 2009). For instance, there is a more-prominent N400 response to sentence endings that are semantically incongruent (relative to congruent) with the rest of the sentence (Kutas \& Hillyard, 1980). Furthermore, N400 amplitude is highly inversely correlated with cloze probability, a measure describing a participant's expectancy for a particular target word (Kutas \& Federmeier, 2011). The more unexpected a sentence ending is, the larger the N400 amplitude (e.g., "The bill was due at the 
end of the month [hour]"; Kutas \& Federmeier, 2011). The N400 response therefore is influenced both by the degree of semantic anomaly and by the degree of predictability (Lau, Phillips, \& Poeppel, 2008). Because the N400 reflects violations of expectancies, it is a useful tool to examine how people derive meaning from language. During online language comprehension, knowledge is accessed and expectancies are generated to facilitate understanding. If the N400 is elicited by a word in a sentence, then the specific knowledge that was violated within the sentence must have been accessed by the person interpreting it. Thus, by signifying violations of expectancies, the N400 can be used to study how different types of knowledge are involved in language comprehension.

Whereas early N400 work focused on violations of linguistic knowledge, researchers have since examined expectancy violations beyond traditional semantic incongruencies. Most notably, Hagoort et al. (2004) observed that violations of world knowledge elicit an N400 response. Specifically, Dutch participants demonstrated prominent N400 responses while reading the sentence, "The Dutch trains are white," given that it is well known among the Dutch that their trains are yellow (Hagoort et al., 2004). Moreover, both the semantic violation (e.g., "The Dutch trains are sour") as well as the world-knowledge violation (e.g., "The Dutch trains are white") led to N400 amplitudes that were nearly identical in size (Hagoort et al., 2004). A recent conceptual replication of this study confirmed these findings, with greater N400 amplitudes elicited by semantic violations (e.g., "Journeys are stripy”) and world-knowledge violations (e.g., "Ladybirds are stripy”) than by non-violations (e.g., "Zebras are stripy”; Dudschig, Maienborn, \& Kaup, 2016).

Following the discovery of N400 responses to world-knowledge violations, researchers have explored how the broader context of a situation interacts with the expectancies people 
generate while interpreting a sentence. In addition to shedding light on how different types of knowledge are integrated during language comprehension, these studies provide clues as to what conditions are required for eliciting the N400. For example, when world-knowledge violations are preceded by a discourse context that makes the violation more acceptable, the N400 response is reduced (Hald, Steenbeek-Planting, \& Hagoort, 2007). This pattern also has been found with sentence endings that violate idiosyncratic characterizations, as when a story of a dancing peanut, "crazy about" a woman, elicited larger-amplitude N400 responses when the peanut subsequently was described as "salted" than as "in love" (Nieuwland \& van Berkum, 2006). These findings provide additional evidence that people generate expectancies based on nonlinguistic knowledge. At the same time, some studies have revealed the N400 to be insensitive to information beyond the sentence level. For instance, sentence endings describing behaviors that violated predictions based on personality traits (e.g., "always opens the door for strangers") did not elicit larger-amplitude N400 responses; instead, these expectancy violations elicited a late ERP positivity peaking at 500-600 ms post-stimulus (Bartholow, Fabiani, Gratton, \& Bettencourt, 2001). Related investigations into the processing of spontaneous trait inferences (Van Duynslaeger, Van Overwalle, \& Verstraeten, 2007) and moral judgment (Leuthold, Kunkel, Mackenzie, \& Filik, 2015) have also found expectancy violations to be indicated by a late ERP positivity rather than a larger-amplitude N400 response.

Research on the N400 also has extended to explore how language comprehension is affected by particular beliefs, perceptions, and other social expectancies. In one study, researchers observed more-prominent N400 responses when participants were presented with a statement that was incongruent with their values, such as "I think euthanasia is an acceptable [unacceptable] course of action" (Van Berkum et al., 2009). The N400 response also has been 
observed in the context of stereotypes. In one study, incongruent word pairs (e.g., Men:

Nurturing; Women: Aggressive) elicited significantly larger N400 amplitudes than did congruent word pairs (e.g., Men: Engineer; Women: Secretary; White, Crites Jr., Taylor, \& Corral, 2009). In addition, previous work has shown that having expectations about a speaker leads to increased N400 amplitude when the speaker provides expectancy-incongruent information (Van Berkum, van den Brink, Tesink, Kos, \& Hagoort, 2008). Specifically, this study showed that the phrase, "If I only looked like Britney Spears in her latest video," elicited a larger-amplitude N400 when uttered by a male speaker than by a female speaker (Van Berkum et al., 2008). Finally, a study with Chinese participants found larger N400 responses to conversations in which the speaker used an inappropriate pronoun (e.g., as when a student uses the less respectful second-person pronoun when speaking to a professor) compared to when the speaker used an appropriate pronoun (Jiang, Li, \& Zhou, 2013).

Given the above-reviewed evidence of N400 responses to non-linguistic violations, we examined whether the study of this component can be extended to illuminate understanding of violations of expectancies based on general social knowledge. Previous studies have focused on powerful violations of specific rather than general social expectancies. However, at the most general level, humans are social creatures who want to belong. The fundamental desire to form and maintain social bonds exerts a powerful and widespread influence on a variety of human behaviors, and belongingness is crucial for physical and psychological well-being (Baumeister \& Leary, 1995). One of the ways people facilitate belongingness is by following social norms, or the rules and standards for acceptable social behavior within a group (Cialdini \& Trost, 1998). Social norms contribute to the generation of expectancies because they provide guidelines for how people should behave. For instance, a basic norm regarding human interaction is that one 
should be cordial; impolite behavior is generally considered unacceptable. Research on ostracism has documented the range of negative affective experiences that occur after an event of social exclusion (Williams, 2000). Furthermore, this work found that whereas being included is favorable to being excluded, being over-included does not result in significantly more positive affective experiences than does being included (Williams, 2000). This suggests that people expect a baseline level of decency from others, such as inclusion in a social interaction. A violation of the expectancy, in the form of exclusion, has strong affective consequences, whereas situations that are consistent with the expectancy, such as inclusion and overinclusion, produce similar effects.

Two recent studies have begun to explore the N400 in response to social norm violations. The first study examined semantic violations as well as "white lies," or factually inaccurate statements told to avoid hurting someone's feelings (Moreno, Casado, \& Martin-Loeches, 2016). Participants were presented with multi-sentence stories in which someone is told either a white lie or a blunt truth (e.g., someone prepares a bad meal for their guests, and one guest says "The meat sauce was tasty [overcooked]"; Moreno et al., 2016). The target word "tasty" is a white lie; it is factually inaccurate, but may be expected based on social norms. The target word "overcooked" is a blunt truth; it is factually accurate but impolite. Participants read for comprehension without performing any additional task (Moreno et al., 2016). The authors observed the classic N400 effect to semantic violations (e.g., "The meat sauce was romantic") but found that, overall, blunt truths did not evoke a greater N400 response than did white lies (Moreno et al., 2016).

However, when the authors analyzed data only for sentences moderately or highly biased to white lying, both semantic violations and blunt truths elicited a greater N400 response than did 
white lies (Moreno et al., 2016). Moreover, for sentences highly biased to white lying, cloze probabilities were larger for white lies $(7.1 \%)$ than for blunt truths $(2.7 \%)$, but in sentences moderately biased to white lying, cloze probabilities for white lies and blunt truths did not differ (5.9\% and 5.8\%, respectively). Thus, whereas sentences highly biased to white lying were confounded with cloze probability, this was not the case for moderately biasing sentences. The authors conclude that social norm violations elicited N400 responses in sentences particularly biased to white lying (Moreno et al., 2016).

Another study, however, suggests a different interpretation $(\mathrm{Mu}$, Kitayama, Han, \& Gelfand, 2015). In this study, participants made judgments regarding whether certain behaviors were appropriate given the situation (e.g., cycling). The stimuli consisted of two sentences and were either appropriate (e.g., "Jacob is in the bike lane"), weak violations (e.g., "Jacob is on the highway"), or strong violations (e.g., "Jacob is on the city sidewalk") given the situation (e.g., "He is cycling"; Mu et al., 2015). Unlike the previous study in which the target word differed across conditions (Moreno et al., 2016), these stimuli control for word-based effects by keeping the target sentence (e.g., "He is cycling") constant across conditions. The authors found a greater N400 response to strong and weak violations compared to appropriate situations (Mu et al., 2015). These results diverge from the previous study in two ways. First, Moreno et al. (2016) did not observe an overall N400 response to their social violations, whereas Mu et al. (2015) did. Furthermore, Mu et al. (2015) reported that both the strong and weak violations elicited a greater N400 response compared to the appropriate condition. This is in contrast with the previous study, which only found greater N400 amplitudes for the strongest violations, but not the weakest violations (Moreno et al., 2016). 
The aim of the present study is to explore the sensitivity of the N400 to violations of social norms. Given previous work on ostracism, which suggests that people expect a baseline level of decency from others (Williams, 2000), this study specifically looked at courteousness in social situations involving the self. By focusing on a specific social norm, this study differs from the work by Mu et al. (2015), which examined the appropriateness of various social behaviors. Moreover, whereas the study by Moreno et al. (2016) looked at the social expectation to be polite but untruthful with white lies, the scenarios did not involve the self. Thus, violations of basic standards of amicable treatment in social situations involving the self may be considered more fundamental violations because they represent a threat to one's sense of belongingness. Generic violations of appropriate behavior, on the other hand, are somewhat ambiguous in that they do not hone in on a basic social norm. We hypothesized that sentences describing situations incongruent (relative to congruent) with basic standards of amicable treatment by others would elicit a larger-amplitude N400. To examine the N400 component, we assessed participants' responses to sentences varying along two dimensions: a social-dimension and a semanticdimension. For the social-dimension, sentence endings were either congruent or incongruent with basic standards of amicable treatment by others (e.g., "Your friend breaks your computer and then apologizes [laughs]"). For the semantic-dimension, sentences contained either a semantically congruent or incongruent word (e.g., "You want to know if your friend wants to go to dinner, so your send your friend an email [a tomato]").

\section{Method}

\section{Participants}


Twenty Stony Brook University undergraduates (15 males), aged 18-27 $(M=20.00)$, participated. Due to experimenter error, there was no recording of the sentence-ratings of one participant.

\section{Procedure}

Participants viewed statements presented one word at a time on a computer monitor (with $75 \mathrm{~Hz}$ refresh rate). There was a blank screen separating the words, and the words were presented centrally. Following Otten and Van Berkum (2007), presentation times were determined individually for each word, based on natural reading times (e.g., Haberlandt \& Graesser, 1985; Legge, Ahn, Klitz, \& Luebker, 1997) and on a subjective evaluation by the experimenter of whether each word was presented for an appropriate length of time. The mean duration of presentation time was $386.59 \mathrm{~ms}$. After each sentence was presented, participants indicated whether they could (coded " 1 ") or could not (coded " 0 ") easily imagine the events it described actually occurring, using a two-key response device. These binary imaginability judgments were used to ensure that participants' natural responses to the stimuli matched the intended manipulation. Asking participants to report whether the events are easily imaginable was preferred over directly asking whether the events are appropriate (cf. Mu et al., 2015), as the latter requires participants to reflect more deliberately. As with Moreno et al. (2016), we did not want to influence how participants processed our stimuli by explicitly asking them to rate the appropriateness of events in which they were not treated amicably. Since the social-dimension sentences described situations involving the self, asking whether the events are easily imaginable provided a natural way to assess whether the events were considered violations of social norms. At the end of the experiment, participants completed Rosenberg's (1965) 10-item measure of self-esteem and Watson, Clark, and Tellegen's (1988) 10-item measures of positive affect and 
negative affect; responses to these two questionnaires were recorded in the event that we or other researchers conducted further experiments with these methods, such that a sufficient cumulative sample size would exist for examining individual-level variation in any effects obtained.

Stimuli. For sentences varying along a semantic-dimension, incongruent terminal words were semantically incongruent with the rest of the sentence (e.g., "You ask the waitress for a glass of shoes"), and congruent terminal words were semantically congruent with the rest of the sentence (e.g., "You ask the waitress for a glass of water"). There were 106 unique pairs of semantic-dimension sentences, with the members of each pair differing only in their terminal words. The sentence stems were presented in randomly varying orders, and provision of each sentence's congruent or incongruent terminal word was determined randomly. For the sentences varying on a social-dimension, incongruent terminal words yielded descriptions of events in which one is not treated amicably (e.g., "You ask someone to coffee and that person says no"), and congruent terminal words yielded descriptions of events in which one is treated amicably (e.g., "You ask someone to coffee and that person says yes"). There were 106 unique pairs of these social-dimension sentences, with the members of each pair differing only in their terminal words. Table 1 provides mean length, frequency, and orthographic neighborhood size for terminal words, as well as latent semantic analysis (LSA) information between the sentence stems and terminal words (Landauer, Foltz, \& Laham, 1998). The sentences varying on a semantic-dimension had approximately the same number of words $(M=13.74 ; S D=2.74)$ as did the sentences varying on a social-dimension $(M=13.82 ; S D=2.69)$. The two sentence types were presented in separate blocks in counter-balanced orders, such that participants viewed 106 semantic-dimension sentences and 106 social-dimension sentences for a total of 212 sentences (see Appendix for list of social-dimension sentences). 


\section{EEG Recording and ERP Analysis}

The continuous EEG was recorded using a 32-channel electrode cap (Compumedics Neuroscan, Charlotte, NC) using a fronto-central electrode as ground and electronically linked mastoid electrodes as reference. The horizontal electrooculogram (EOG) was monitored from electrodes at the outer canthi of the eyes, and vertical EOG was monitored from electrodes above and below the orbital region of the left eye. Impedances for all electrodes were kept below 10 $\mathrm{k} \Omega$. The EEG and EOG signals were digitized at $500 \mathrm{~Hz}$ and amplified with a gain of 1000 . The filter bandpass was .01-30 Hz. The continuous data were divided into epochs beginning $100 \mathrm{~ms}$ before each sentence's terminal word was presented and concluding $900 \mathrm{~ms}$ thereafter. Mean amplitude during the first $100 \mathrm{~ms}$ of each epoch served as a baseline that was subtracted from amplitude measurements at remaining time points of that epoch. Using independent component analysis (ICA) via the Runica function of EEGlab (Delorme \& Makeig, 2004), components reflecting ocular artifacts were identified and subtracted. Following ICA-based corrections, any remaining epochs with EEG voltages exceeding $+/-75 \mu \mathrm{V}$ at any channel were rejected from the average (resulting in exclusion of $3.66 \%$ of remaining trials). For the social-dimension sentences, the number of trials remaining after artifact exclusion ranged from 37-60 (Median $=52.5)$ for congruent sentences and 42-61 (Median = 52) for incongruent sentences. For the semanticdimension sentences, the number of trials remaining after artifact exclusion ranged from 40-70 $($ Median $=52)$ for congruent sentences and 36-65 (Median = 49.5) for incongruent sentences. Based on the previous literature, N400 amplitude was defined as mean amplitude between 300 and $500 \mathrm{~ms}$ following stimulus presentation (Lau et al., 2008). Because no clear laterality differences emerged (consistent with Hagoort et al., 2004), we limited statistical analyses to data from the midline electrodes FZ, FCZ, CZ, CPZ, and PZ. As recommended by Nieuwenhuis, 
Forstmann, and Wagenmakers (2011), to assess whether the magnitude of the effect of the congruency manipulations on N400 amplitude differed significantly across the social and semantic-dimension sentence types, we used analysis of variance (ANOVA) to test whether sentence type (social vs. semantic) interacted with congruency (congruent vs. incongruent) to affect N400 amplitude. Greenhouse-Geisser corrections to degrees of freedom were applied to ANOVAs with more than two within-participant levels (Greenhouse \& Geisser, 1959).

\section{Results}

Table 2 provides the imaginability judgments for both sentence types. Imaginability judgments were analyzed in a 2 (Sentence Type: social-dimension vs. semantic-dimension) x 2 (Congruency: congruent vs. incongruent) repeated measures ANVOA. There was a significant main effect of congruency, $F(1,18)=181.67, p<.0001$, partial $\eta^{2}=.91$. This effect was moderated by sentence type, $F(1,18)=23.67, p<.0001$, partial $\eta^{2}=.59$. Follow-up analyses found that incongruently terminated sentences were judged significantly more difficult to imagine occurring than congruently-terminated sentences, for both the semantic-dimension sentences $\left(M_{\text {difference }}=0.77 ; S D_{\text {difference }}=0.24 ; t(18)=14.08, p<.0001\right)$ and the social-dimension sentences $\left(M_{\text {difference }}=0.50 ; S D_{\text {difference }}=0.24 ; t(18)=9.26, p<.0001\right)$. Furthermore, among incongruently terminated sentences, semantic-dimension sentences were judged significantly more difficult to imagine occurring than were social-dimension sentences, $t(18)=-6.02, p<$ .0001 .

Mean amplitudes during the N400 window (see Table 3) were analyzed in a 2 (Sentence Type: social-dimension vs. semantic-dimension) x 2 (Congruency: congruent vs. incongruent) $\mathrm{x}$ 5 (Electrode: FZ, FCZ, CZ, CPZ, or PZ) repeated-measures ANOVA. Most importantly, there was a significant interaction between sentence type and congruency, $F(1,19)=7.86, p<.02$, 
partial $\eta^{2}=.29$, which was moderated further by electrode location, $F(4,76)=6.65, p<.01$, partial $\eta^{2}=.26$. This interaction was not moderated by the order in which the social and semantic-dimension sentences were presented, $F(4,72)=1.29, p=.28$, partial $\eta^{2}=.07$. To clarify the nature of the Sentence Type x Congruency x Electrode interaction, we conducted follow-up 2 (Congruency: congruent vs. incongruent) x 5 (Electrode: FZ, FCZ, CZ, CPZ, or PZ) repeated-measures ANOVAs separately for each sentence type. Figure 1 shows the grandaverage ERP waveforms for social and semantic-dimension sentences, and Figure 2 shows the topographic distributions of the N400. For the semantic-dimension sentences, there was a significant main effect of congruency, $F(1,19)=9.81, p<.01$, partial $\eta^{2}=.34$, and a significant interaction between congruency and electrode location, $F(4,76)=3.83, p=.03$, partial $\eta^{2}=.17$, consistent with previous findings of maximal N400 effects at centro-parietal electrodes (e.g., Hagoort et al., 2004). To analyze the significant ANOVA result, follow-up $t$ tests were conducted at each electrode site. To correct for multiple comparisons, the alpha level was adjusted using Holm's sequential Bonferroni method (Holm, 1979). The post-hoc tests indicate that the effect of congruency for the semantic-dimension sentences was significant at each electrode site $(\mathrm{FZ}: t(19)=2.13, p=.046 ; \mathrm{FCZ}: t(19)=2.70, p=.028 ; \mathrm{CZ}: t(19)=3.13, p=$ .022 ; CPZ: $t(19)=3.00, p=.022 ; \mathrm{PZ}: t(19)=3.94, p=.005)$. In contrast, for the socialdimension sentences, there was no significant main effect of congruency, $F(1,19)=0.67, p=$ .42 , partial $\eta^{2}=.03$, and no significant interaction between congruency and electrode location, $F(4,76)=1.70, p=.20$, partial $\eta^{2}=.08$.

To address the possibility that the observed N400 effects for semantic but not socialdimension sentences might have reflected differences in the congruency manipulations' impacts on imaginability of the events described in the social vs. semantic sentences, we next performed 
follow-up analyses on the subset of trials in which participants' imaginability judgments matched the intended congruence conditions (i.e., when participants indicated they could easily imagine the events occurring for congruent sentences and could not easily imagine the events occurring for incongruent sentences). Because behavioral data were missing for one participant, these analyses were limited to 19 participants. For the social-dimension sentences, the number of trials remaining after artifact exclusion ranged from 19-55 (Median $=42.3$ ) for congruent sentences and 13-57 (Median = 42) for incongruent sentences. For the semantic-dimension sentences, the number of trials remaining after artifact exclusion ranged from 30-65 (Median = 45.6) for congruent sentences and 19-61 (Median = 50.5) for incongruent sentences. Mean amplitudes during the N400 window (see Table 4) were analyzed in a 2 (Sentence Type: social-dimension vs. semantic-dimension) x 2 (Congruency: congruent vs. incongruent) x 5 (Electrode: FZ, FCZ, $\mathrm{CZ}, \mathrm{CPZ}$, or PZ) repeated-measures ANOVA. There was a significant interaction between congruency and sentence type, $F(1,18)=11.30, p<.01$, partial $\eta^{2}=.39$, which was not moderated further by electrode location, $F(4,72)=2.80, p=.08$, partial $\eta^{2}=.13$. Follow-up 2 (Congruency: congruent vs. incongruent) x 5 (Electrode: FZ, FCZ, CZ, CPZ, or PZ) repeatedmeasures ANOVAs were conducted separately for each sentence type. For trials where imaginability judgments matched sentence congruency, Figure 3 shows the grand-average ERP waveforms for social and semantic-dimension sentences, and Figure 4 shows the topographic distributions of the N400. For the semantic-dimension sentences, there was a significant main effect of congruency, $F(1,18)=12.16, p<.01$, partial $\eta^{2}=.40$, whereas the interaction between congruency and electrode location was not significant, $F(4,72)=2.98, p=.06$, partial $\eta^{2}=.14$. For the social-dimension sentences, there was no significant interaction between congruency and 
electrode location, $F(4,72)=0.57, p=.55$, partial $\eta^{2}=.03$. Echoing the above-reported results, the main effect of congruency also was not significant, $F(1,18)=0.23, p=.64$, partial $\eta^{2}=.01$.

\section{Discussion}

The present work explored the sensitivity of the N400 to violations of expectancies based on general social knowledge. Our findings of N400 modulation by semantic but not social norm violations help clarify potential boundary conditions for eliciting the N400. These results are partially consistent with the study by Moreno et al. (2016), in which the original analyses found that social norm violations in the form of "blunt truths," which are accurate but impolite statements, did not elicit an enhanced N400 (relative to "white lies"). The authors did find a significant N400 response to social norm violations after limiting their analyses to scenarios that a separate sample of participants rated as particularly biased to white lying (Moreno et al., 2016). However, the current study did not find an N400 response to social norm violations, even after limiting analyses to trials in which participants' imaginability judgments matched the intended manipulation. Furthermore, these findings are in contrast with the study by Mu et al. (2015), which found that both strong and weak social norm violations elicited an N400 response.

One explanation for these inconsistent findings could be related to the stimuli, which differed across experiments. For example, stimuli were one sentence long in the current study, but were two or more sentences in the studies by Moreno et al. (2016) and Mu et al. (2015). Based on previous evidence that the N400 response is modulated by information from multiple sentences (e.g., Hald et al., 2007), it is possible that the additional information builds a broader context, making the violations in the Moreno et al. (2016) and Mu et al. (2015) studies more salient. Another feature of the stimuli in the current study concerns the presence of yes-no particles in approximately one-fifth of the social-dimension sentences compared to none 
occurring in the semantic-dimension sentences. Although the yes-no sentence endings were equally distributed among the congruent and incongruent social-dimension sentences (i.e., 11 congruent sentences ended with "yes" and 11 incongruent sentences ended with "no"), the presence of yes-no particles in the social but not the semantic-dimension sentences introduces variation in these conditions. Future work is needed to determine the influence of yes-no particles on the N400 response.

In the current study, participants judged sentences describing themselves treated nonamicably by others to be vastly less subjectively imaginable than sentences describing themselves treated amicably by others (Cohen's $d=2.08$ ). Nevertheless, there were no differences in N400 amplitude between congruent and incongruent social-dimension sentences. This finding may appear surprising given the high inverse correlation of N400 amplitude with cloze probability (Lau et al., 2008). However, in their study on the interaction of discourse context and world knowledge violations, Hald et al. (2007) found that N400 amplitude did not always correlate with cloze probability. In particular, when the world knowledge violation was embedded in a discourse context that made the violation more acceptable, congruent and incongruent sentences elicited N400 amplitudes that were indistinguishable, despite differing cloze ratings (28.12\% and 58.37\%; Hald et al., 2007). Therefore, the results of that study suggest that the N400 is not simply a physiological measure of expectancy violations. Nevertheless, future work may benefit from investigating relations between cloze probability, which was not assessed in the present work, and imaginability, which was assessed in this work.

Although the violations of social norms conveyed in the present investigation's socialincongruent sentence-endings were unexpected, they appear to have been sufficiently comprehensible such that they did not require the enhanced contextual integration that typically 
is associated with enhanced N400 amplitude. In a similar vein, the use of an honest but impolite truth in place of a more socially acceptable white lie (Moreno et al., 2016) is unexpected, but still not difficult to comprehend, considering that people do not always tell white lies to avoid blunt truths. However, the social norm violations used by Mu et al. (2015) differ in that they appear to require the most efforts toward comprehension. For instance, while some stimuli describe inappropriate social behavior (e.g., "Steve is in the library. He is yelling"), others describe behavior that is closer to random than inappropriate (e.g., "John is at a funeral. He is applauding"; "Mary is at a class lecture. She is crying"; "Claire is at church. She is bargaining"). These situations appear to violate more than social norms; they also appear to violate basic assumptions of meaning in these contexts. Similar to Kutas and Hillyard's (1980) classic semantic violation "I take my coffee with cream and dog," some of the stimuli from Mu et al. (2015) may be difficult to comprehend because they require extensive comprehension. From this standpoint, the present results appear consistent with the view that enhanced N400 responses should be associated not simply with text depicting globally unexpected events but rather with text requiring enhanced contextual integration in order to be comprehended.

It is possible that the absent N400 effect to social norm violations in the present study might be due to component overlap with a late positive potential. Previous research on spontaneous trait inferences have found that critical sentences inconsistent with a previously established personality trait do not modulate the N400, but trigger a positivity peaking around 500-600 ms post-stimulus (Bartholow et al., 2001; Leuthold et al., 2015; Van Duynslaeger et al., 2007). This absent N400 effect is suggested to be due to the late positive potential obscuring the N400 response (Baetens, der Cruyssen, Achtziger, Vandekerckhove, \& Van Overwalle, 2011). However, social norm violations have been shown to modulate the N400 in previous work 
(Moreno et al., 2016; Mu et al., 2015). In the case of Moreno et al. (2016), the N400 response and a later frontal positivity were seen to social norm violations, indicating that component overlap did not wash out the N400 effect. In the present study, incongruent social-dimension sentences elicited a slightly larger positivity than congruent social-dimension sentences, mainly over centro-parietal electrodes, but this effect was not statistically significant. Given that social violations are expected to result in smaller N400 effects than semantic violations, this study may be limited by statistical power. Future research with more participants would provide more precise measurements of these effects.

Taken together, the results from the present study did not suggest that the N400 is generated by general social-expectancy violations. Despite observing the classic N400 effect to semantic incongruences, violations of general social norms did not elicit the N400 response. Further investigation of the conditions under which violations of social knowledge elicit N400 responses can help further clarify the psychological processes through which people resolve those information-processing conflicts. 


\section{References}

Baetens, K., der Cruyssen, L. V., Achtziger, A., Vandekerckhove, M., \& Van Overwalle, F. (2011). N400 and LPP in spontaneous trait inferences. Brain Research, 1418, 83-92. doi: 10.1016/j.brainres.2011.08.067

Bartholow, B. D., Fabiani, M., Gratton, G., \& Bettencourt, B. A. (2001). A psychophysiological analysis of cognitive processing of and affective responses to social expectancy violations. Psychological Science, 12(3), 197-204. https://doi.org/10.1111/14679280.00336

Baumeister, R. F., \& Leary, M. R. (1995). The need to belong: Desire for interpersonal attachments as a fundamental human motivation. Psychological Bulletin, 117(3), 497529. http://dx.doi.org/10.1037/0033-2909.117.3.497

Brown, C., \& Hagoort, P. (1993). The processing nature of the n400: Evidence from masked priming. Journal of Cognitive Neuroscience, 5(1), 34-44. doi:10.1162/jocn.1993.5.1.34

Cialdini, R. B., \& Trost, M. R. (1998). Social influence: Social norms, conformity, and compliance. In D. T. Gilbert, S. T. Fiske, \& G. Lindzey (Eds.), The handbook of social psychology (4th ed., Vol. 2, pp. 151-192). Boston: McGraw-Hill.

Delorme, A., \& Makeig, S. (2004). EEGLAB: An open source toolbox for analysis of single-trial EEG dynamics including independent component analysis. Journal of Neuroscience Methods, 134(1), 9-21. doi:10.1016/j.jneumeth.2003.10.009

Dudschig, C., Maienborn, C., \& Kaup, B. (2016). Is there a difference between stripy journeys 
and stripy ladybirds? The N400 response to semantic and world-knowledge violations during sentence processing. Brain and Cognition, 103, 38-49. doi:10.1016/j.bandc.2016.01.001

Greenhouse, S. W., \& Geisser, S. (1959). On methods in the analysis of profile data. Psychometrika, 24(2), 95-112. doi:10.1007/bf02289823

Haberlandt, K., \& Graesser, A. C. (1985). Component processes in text comprehension and some of their interactions. Journal of Experimental Psychology: General, 114(3), 357-374. doi:10.1037/0096-3445.114.3.357

Hagoort, P., Hald, L., Bastiaansen, M., \& Petersson, K. M. (2004). Integration of world meaning and world knowledge in language comprehension. Science, 304(5669), 438-441. doi:10.1126/science.1095455

Hald, L. A., Steenbeek-Planting, E. G., \& Hagoort, P. (2007). The interaction of discourse context and world knowledge in online sentence comprehension. Evidence from the N400. Brain Research, 1146, 210-218. doi:10.1016/j.brainres.2007.02.054

Holm, S. (1979). A simple sequentially rejective multiple test procedure. Scandinavian Journal of Statistics, 6(2), 65-70. Retrieved from http://www.jstor.org/stable/4615733

Jiang, X., Li, Y., \& Zhou, X. (2013). Is it over-respectful or disrespectful? Differential patterns of brain activity in perceiving pragmatic violation of social status information during utterance comprehension. Neuropsychologia, 51(11), 2210-2223. doi:10.1016/j.neuropsychologia.2013.07.021

Kutas, M., \& Federmeier, K. D. (2011). Thirty years and counting: Finding meaning in the N400 component of the event-related brain potential (ERP). Annual Review of Psychology, 62, 621-647. doi:10.1146/annurev.psych.093008.131123 
Kutas, M., \& Hillyard, S. A. (1980). Reading senseless sentences: Brain potentials reflect semantic incongruity. Science, 207(4427), 203-203. doi: 10.1126/science.7350657

Landauer, T. K., Foltz, P. W., \& Laham, D. (1998). An introduction to latent semantic analysis. Discourse Processes, 25(2-3), 259-284. doi: 10.1080/01638539809545028

Lau, E. F., Phillips, C., \& Poeppel, D. (2008). A cortical network for semantics: (De)constructing the N400. Nature Reviews Neuroscience, 9(12), 920-933. doi:10.1038/nrn2532

Legge, G. E., Ahn, S. J., Klitz, T. S., \& Luebker, A. (1997). Psychophysics of reading. XVI. The visual span in normal and low vision. Vision Research, 37(14), 1999-2010. https://doi.org/10.1016/S0042-6989(97)00017-5

Leuthold, H., Kunkel, A., Mackenzie, I. G., \& Filik, R. (2015). Online processing of moral transgressions: ERP evidence for spontaneous evaluation. Social Cognitive and Affective Neuroscience, 10, 1021-1029. http://dx.doi.org/10.1093/scan/nsu151.

Lund, K., \& Burgess, C. (1996). Producing high-dimensional semantic space from lexical cooccurrence. Behavior Research Methods, 28(2), 203-208. doi:10.3758/BF03204766

Moreno, E. M., Casado, P., \& Martin-Loeches, M. (2016). Tell me sweet little lies: An eventrelated potentials study on the processing of social lies. Cognitive, Affective, \& Behavioral Neuroscience, 16(4), 616-625. doi:10.3758/s13415-016-0418-3

Mu, Y., Kitayama, S., Han, S., \& Gelfand, M. (2015). How culture gets embrained: Cultural differences in event-related potentials of social norm violations. Proceedings of the National Academy of Sciences, 112(50), 15348-15353. doi:10.1073/pnas.1509839112

Nieuwenhuis, S., Forstmann, B. U., \& Wagenmakers, E. J. (2011). Erroneous analyses of interactions in neuroscience: A problem of significance. Nature Neuroscience, 14(9), 1105-1107. doi: 10.1038/nn.2886 
Nieuwland, M. S., \& van Berkum, J. J. A. (2006). When peanuts fall in love: N400 evidence for the power of discourse. Journal of Cognitive Neuroscience, 18(7), 1098-1111. doi: 10.1162/jocn.2006.18.7.1098

Otten, M., \& Van Berkum, J. J. (2007). What makes a discourse constraining? Comparing the effects of discourse message and scenario fit on the discourse-dependent N400 effect. Brain Research, 1153, 166-177. doi:10.1016/j.brainres.2007.03.058

Rosenberg, M. (1965). Society and the adolescent self-image. Princeton, NJ: Princeton University Press.

Van Berkum, J. J., Holleman, B., Nieuwland, M., Otten, M., \& Murre, J. (2009). Right or wrong? The brain's fast response to morally objectionable statements. Psychological Science, 20(9), 1092-1099. doi:10.1111/j.1467-9280.2009.02411.x

Van Berkum, J. J., van den Brink, D., Tesink, C. M., Kos, M., \& Hagoort, P. (2008). The neural integration of speaker and message. Journal of Cognitive Neuroscience, 20(4), 580-591. doi:10.1162/jocn.2008.20054

Van Duynslaeger, M., Van Overwalle, F., \& Verstraeten, E. (2007). Electrophysiological time course and brain areas of spontaneous and intentional trait inferences. Social Cognitive and Affective Neuroscience, 2, 174-188. doi:10.1093/scan/nsm016

Watson, D., Clark, L. A., \& Tellegen, A. (1988). Development and validation of brief measures of positive and negative affect: The PANAS scales. Journal of Personality and Social Psychology, 54(6), 1063-1070. doi: 10.1037//0022-3514.54.6.1063

White, K. R., Crites, S. L., Jr., Taylor, J. H., \& Corral, G. (2009). Wait, what? Assessing stereotype incongruities using the N400 ERP component. Social Cognitive and Affective Neuroscience, 4(2), 191-198. doi:10.1093/scan/nsp004 
Williams, K. D., Cheung, C. K. T., \& Choi, W. (2000). Cyberostracism: Effects of being ignored over the internet. Journal of Personality and Social Psychology, 79(5), 748-762. doi: $10.1037 / 0022-3514.79 .5 .748$

Tables and Figures

Table 1.

Mean Length, Frequency, Orthographic Neighborhood Size, and LSA information (SD in parentheses) for Terminal Words

\begin{tabular}{llllll}
\hline \hline Trial & Congruency & Length & $\begin{array}{l}\text { HAL Log } \\
\text { Frequency }\end{array}$ & $\begin{array}{l}\text { Orthographic } \\
\text { neighborhood size }\end{array}$ & $\begin{array}{l}\text { LSA } \\
\text { information }\end{array}$ \\
\hline Semantic & Congruent & $5.52(1.98)$ & $9.22(1.59)$ & $6.65(7.06)$ & $0.14(0.12)$ \\
& Incongruent & $5.53(1.90)$ & $9.12(1.67)$ & $6.33(6.72)$ & $0.03(0.07)$ \\
\hline Social & Congruent & $5.82(2.22)$ & $10.65(1.88)$ & $5.11(6.24)$ & $0.04(0.08)$ \\
& Incongruent & $5.67(2.10)$ & $10.63(1.91)$ & $5.81(6.56)$ & $0.03(0.07)$ \\
\hline
\end{tabular}

Note. HAL = Hyperspace Analogue to Language (Lund \& Burgess, 1996); LSA = latent semantic analysis. 
Table 2.

Mean Imaginability Judgments (SD in parentheses) for Semantic and Social Sentence Types

\begin{tabular}{clc}
\hline \hline Trial & Congruency & Rating \\
\hline Semantic & Congruent & $0.84(0.15)$ \\
& Incongruent & $0.07(0.13)$ \\
\hline Social & Congruent & $0.82(0.15)$ \\
& Incongruent & $0.32(0.22)$ \\
\hline
\end{tabular}


Table 3.

Mean N400 Amplitudes (in $\mu V$; SD in parentheses) in the 300-500 ms Time Window for All Trials

\begin{tabular}{cllll}
\hline \hline Trial & Channel & Congruent & Incongruent & Difference \\
\hline Semantic & FZ & $2.18(2.59)$ & $0.88(2.48)$ & $1.30(2.74)$ \\
& FCZ & $2.19(3.02)$ & $0.35(3.13)$ & $1.84(3.05)$ \\
& CZ & $2.07(3.14)$ & $-0.24(3.38)$ & $2.31(3.42)$ \\
& CPZ & $2.42(2.96)$ & $0.12(3.32)$ & $2.30(3.42)$ \\
& PZ & $2.99(2.78)$ & $0.53(3.05)$ & $2.47(2.80)$ \\
\hline Social & FZ & $2.93(2.43)$ & $3.01(2.72)$ & $-0.08(2.48)$ \\
& FCZ & $3.09(2.62)$ & $3.30(3.01)$ & $-0.22(2.70)$ \\
& CZ & $2.77(2.78)$ & $3.31(2.83)$ & $-0.54(2.53)$ \\
& CPZ & $3.17(2.60)$ & $3.68(2.74)$ & $-0.51(2.10)$ \\
& PZ & $3.27(2.54)$ & $3.94(2.84)$ & $-0.67(1.71)$ \\
\hline
\end{tabular}


Table 4.

Mean N400 Amplitudes (in $\mu V$; SD in parentheses) in the 300-500 ms Time Window for Trials Where Imaginability Judgments Matched Sentence Congruency

\begin{tabular}{clccc}
\hline \hline Trial & Channel & Congruent & Incongruent & Difference \\
\hline Semantic & FZ & $2.41(2.90)$ & $0.69(2.62)$ & $1.72(3.03)$ \\
& FCZ & $2.48(3.22)$ & $0.27(3.30)$ & $2.22(3.24)$ \\
& CZ & $2.31(3.16)$ & $-0.36(3.49)$ & $2.66(3.38)$ \\
& CPZ & $2.48(2.84)$ & $-0.13(3.39)$ & $2.61(3.42)$ \\
& PZ & $3.04(2.67)$ & $0.26(3.05)$ & $2.78(2.64)$ \\
\hline Social & FZ & $2.92(2.38)$ & $2.91(2.96)$ & $0.01(2.48)$ \\
& FCZ & $3.16(2.55)$ & $3.35(3.37)$ & $-0.18(2.41)$ \\
& CZ & $2.89(2.80)$ & $3.32(3.34)$ & $-0.43(2.36)$ \\
& CPZ & $3.44(2.56)$ & $3.58(3.16)$ & $-0.14(1.94)$ \\
& PZ & $3.40(2.54)$ & $3.76(3.35)$ & $-0.36(1.69)$ \\
\hline
\end{tabular}



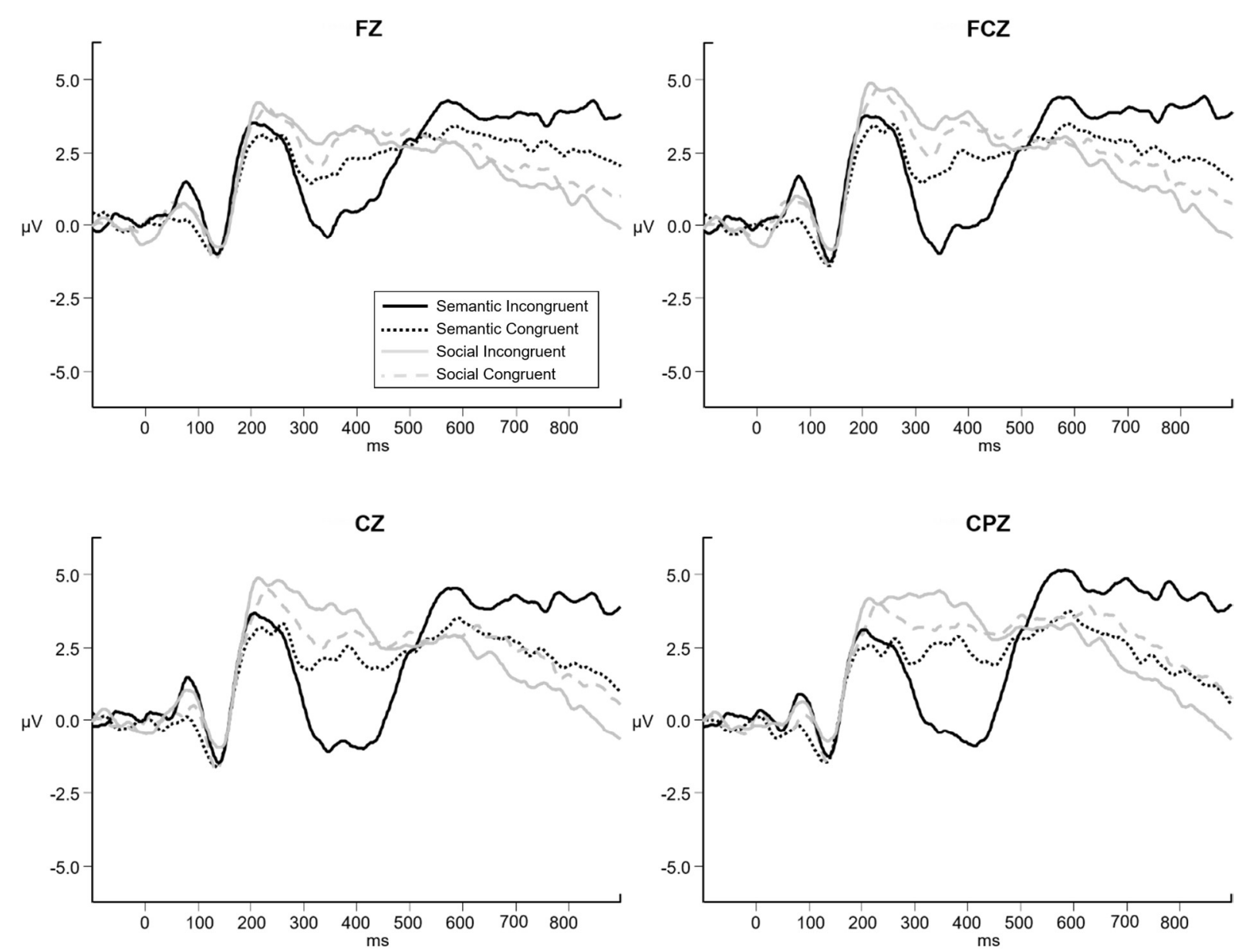


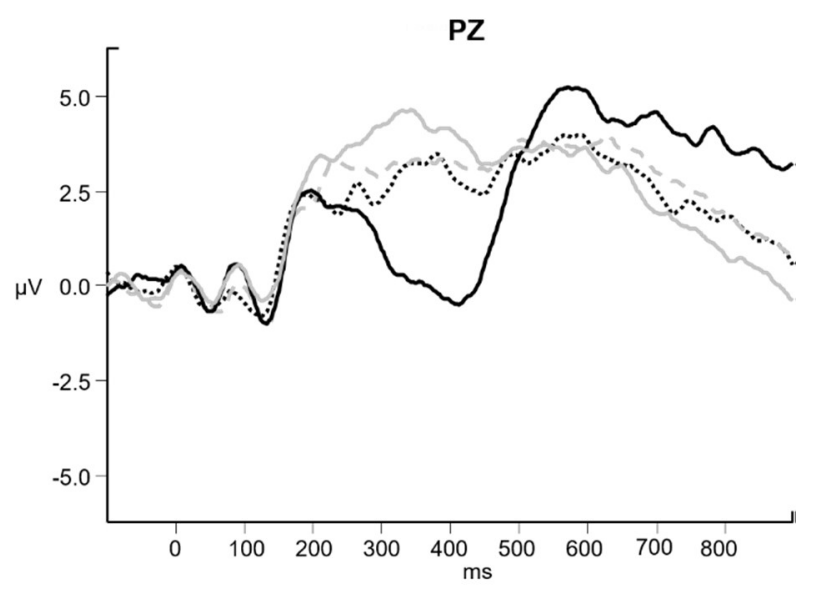

Figure 1. Grand-average ERP waveforms at electrode sites FZ, FCZ, CZ, CPZ, and PZ for social and semantic-dimension sentences, separated by congruency.

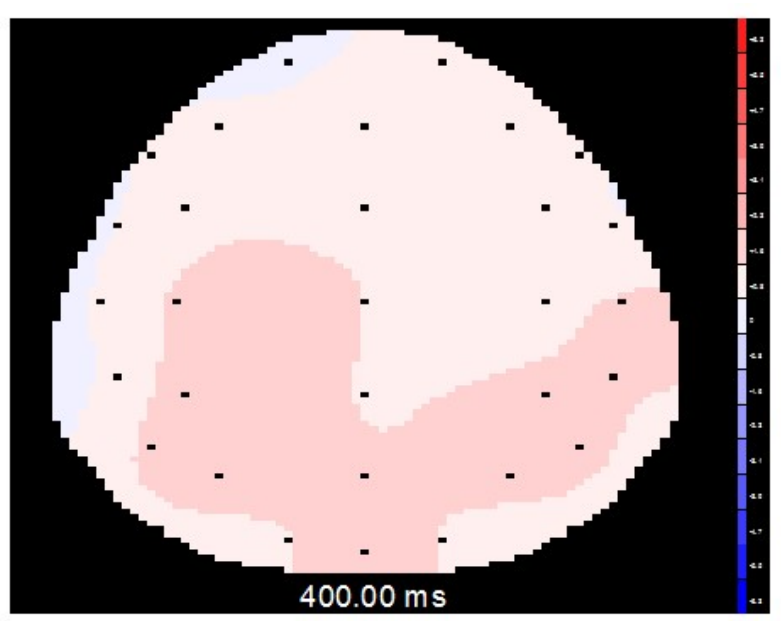

Social

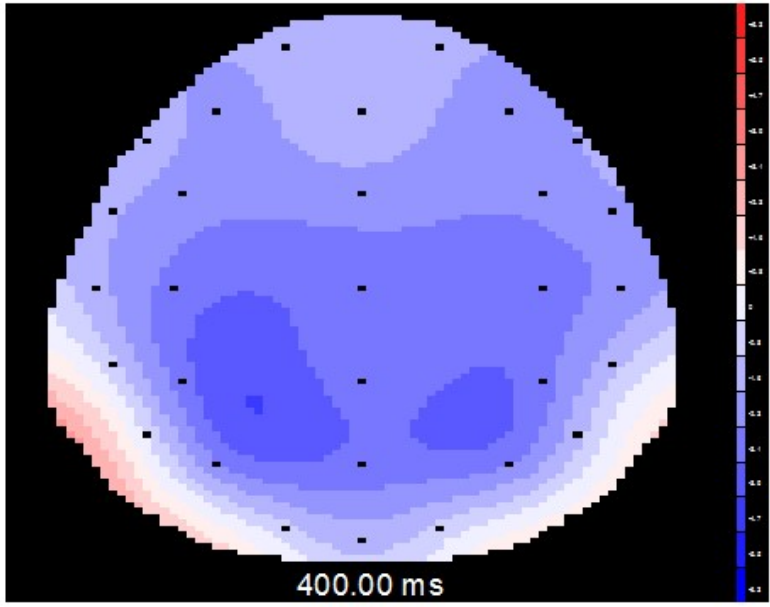

Semantic

Figure 2. Topographic distributions of the N400 for the social and semantic-dimension sentences calculated as the difference between incongruent and congruent conditions at $400 \mathrm{~ms}$. 

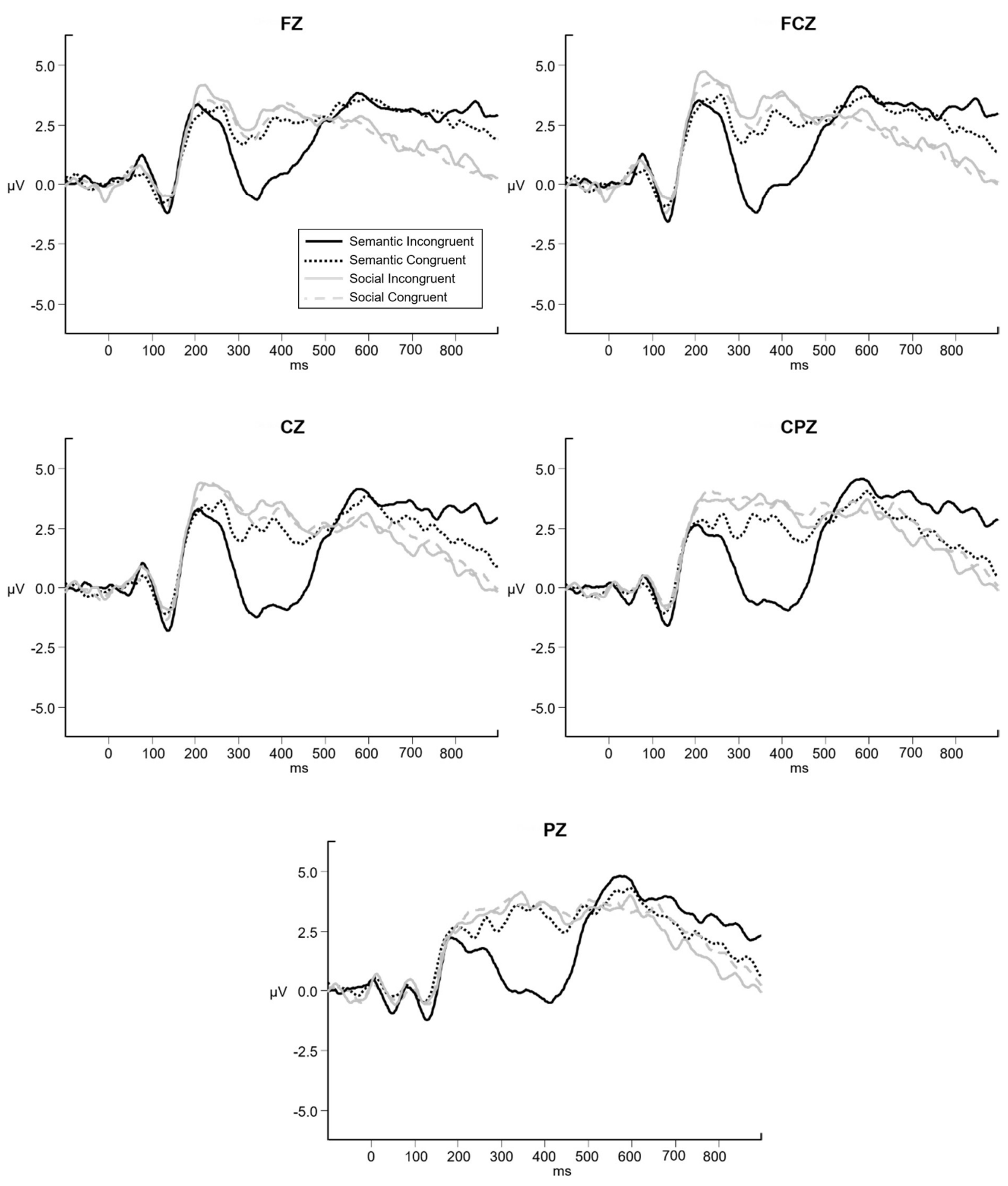
Figure 3. Grand-average ERP waveforms at electrode sites FZ, FCZ, CZ, CPZ, and PZ for social and semantic-dimension sentences, separated by congruency, for trials where imaginability judgments matched sentence congruency.

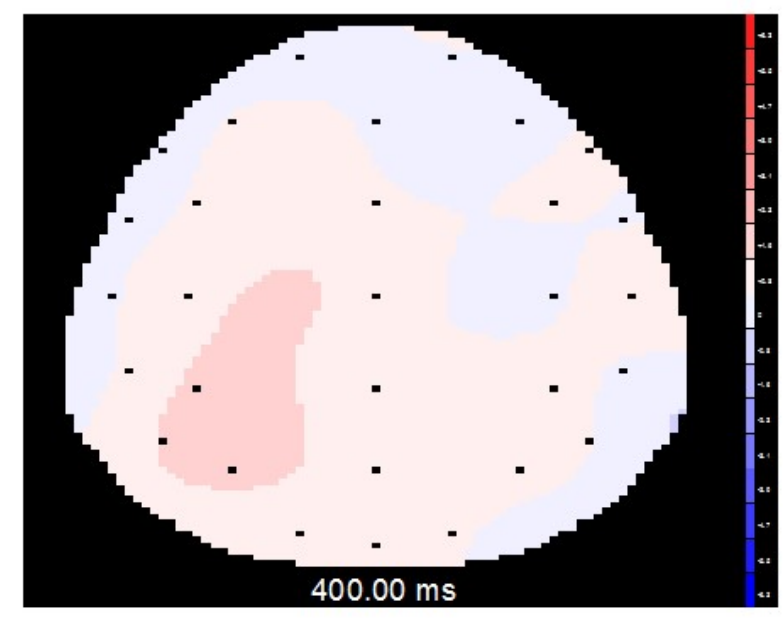

Social

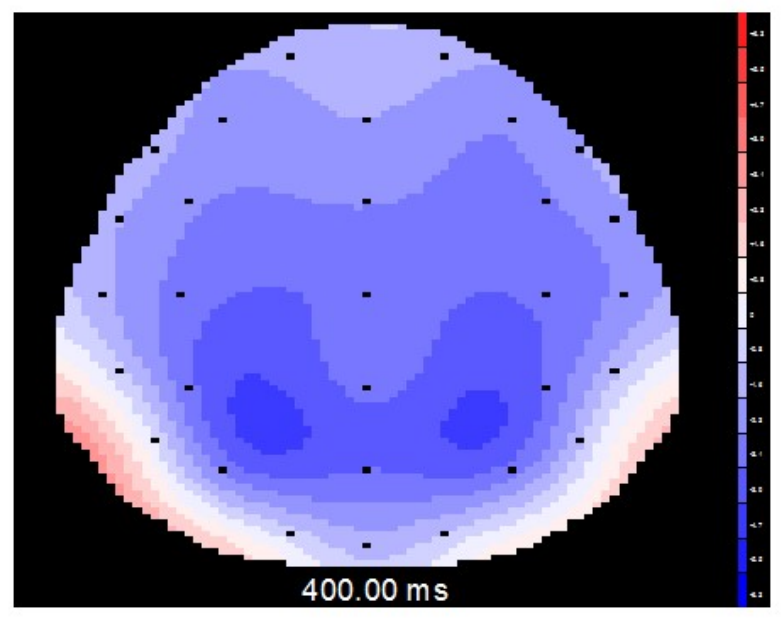

Semantic

Figure 4. Topographic distributions of the N400 for the social and semantic-dimension sentences calculated as the difference between incongruent and congruent conditions at $400 \mathrm{~ms}$, for trials where imaginability judgments matched sentence congruency. 
Appendix

\section{List of Social-Dimension Sentences}

1. You ask someone out and that person says yes [no]

2. Someone is handing out free movie tickets and you are given some [none]

3. Teams are picked for dodge ball and you are picked first [last]

4. You ask a friend to pick up a book for you and your friend says yes [no]

5. You ask someone to coffee and that person says yes [no]

6. You ask to borrow a classmate's notes and your classmate says yes [no]

7. You drop your fork and ask the waiter to bring another one and he does [doesn't]

8. You ask your friend for a ride to the store and your friend agrees [refuses]

9. Your friend makes a list of people to invite to a party and your name is present [absent]

10. You ask to borrow your roommate's stapler and your roommate says yes [no]

11. It is your birthday and your best friend remembers [forgets]

12. A classmate accidentally knocks your books out of your hands and then apologizes [laughs]

13. You arrive at a restaurants and the host seats you at the table that is considered the best [worst]

14. You invite someone to be your friend on myspace and that person accepts [declines]

15. You ask a classmate to work with you on a class project and that person says yes [no]

16. You invite your friends to a party and most of them say yes [no]

17. You ask your roommate to turn down his/her music and he/she does [won't]

18. You ask your friend to call you over the weekend and your friend does [doesn't]

19. You are running for an elevator and someone inside presses the button for door-open [doorclose]

20. A friend with free concert tickets offers you one [none]

21. Some dormmates start a pizza party and ask you to stay [leave]

22. You want to join your friends as they head to a bar and they ask that you do [don't]

23. You apply to join a school club and club members vote yes [no]

24. You plan to meet a friend at the library and your friend remembers [forgets]

25. In the rain, you ask a classmate to share her large umbrella and she agrees [refuses]

26. You ask some acquaintances if they can give you a ride and they say yes [no]

27. You wait in a long line at a nightclub and then the doorman tells you to go inside [home]

28. You ask your friend to return your favorite DVD and your friend does [doesn't]

29. You want to join your friends on a trip they've planned and they ask that you do [don't]

30. You hope to be invited to a weekend party and you are [aren't]

31. You arrive a little late to meet friends and your friends have stayed [left]

32. Your friend offers to buy your computer and offers you a price that is fair [unfair] 
33. You're hungry and ask a friend to share a sandwich and your friend agrees [refuses]

34. You ask someone with a lot of cash to lend you $\$ 5$ and that person says yes [no]

35. You text someone and wait for an answer and the person does [doesn't]

36. You remind your friend to pay you back the $\$ 20$ he borrowed and he pays [refuses]

37. You are on the bus and someone sits next to you and says hello [move]

38. You tell a favorite joke and your friend laughs [yawns]

39. You fall on the ice and your friend gives you a hand [kick]

40. You ace an exam and your friend says the exam must have been difficult [easy]

41. It is your birthday and you receive presents [nothing]

42. The zipper on your jacket is stuck so you ask a friend for assistance and your friend helps [refuses]

43. You go to your professor's office hours with a question and you are helped [ignored]

44. Your friend breaks your computer and then apologizes [laughs]

45. You cancel dinner because you are sick, and your friend is understanding [upset]

46. A friend finds your lost textbook and keeps it to return [sell]

47. You fail an important exam and your closest friend seems worried [happy]

48. You ask a friend to turn in your assignment for you and your friend agrees [refuses]

49. You give a close friend a gift and your friend says it is great [awful]

50. You tell your friend exciting news and your friend seems interested [bored]

51. The professor asks your friend if you cheated and your friend says no [yes]

52. You tell a friend you feel like an idiot and your friend disagrees [agrees]

53. Some classmates want to exclude you from a party and your best friend disagrees [agrees]

54. You ask your friend if you look flabby and your friend says no [yes]

55. You ask a classmate if she's mad at you and your classmate says no [yes]

56. To be polite, you offer to pay the restaurant bill and your wealthy friend refuses [agrees]

57. You tell a friend that you feel like everybody hates you, and your friend disagrees [agrees]

58. Club members vote to kick you out of a club and your best friend votes no [yes]

59. You tell a friend you'll probably fail a class, and your friend disagrees [agrees]

60. An acquaintance says that you're smelly, and your roommates disagree [agree]

61. You ask a friend if you really have to pay back a small loan and your friend says no [yes]

62. You tell a friend that she's way smarter than you and your friend disagrees [agrees]

63. You're nominated as the class's worst dresser, and your best friend votes no [yes]

64. Someone asks your roommate if you are a slob, and your roommate says no [yes]

65. You ask a friend if your haircut looks bad, and your friend says no [yes]

66. You ask your boss if you have to be the one to clean the toilet again, and your boss says no [yes]

67. You ask your friends if they want you to leave, and your friends say no [yes]

68 . You ask your roommate not to eat your last pack of ramen noodles, and your roommate doesn't [does]

69. You ask your friend not to cut you in line again, and your friend doesn't [does]

70. You ask your friend not to call your boyfriend/girlfriend again, and your friend doesn't [does]

71. You ask your roommate not to borrow your jacket, and your roommate doesn't [does] 
72. You find an online list of most ugly Stony Brook students, and your name is absent [present] 73. Your boss makes a list of which days people have to clean the grease trap, and your name is last [first]

74. When offered a chance to kick you out of a chat group, your friends decline [accept] 75. You wonder how many people in the class are smarter than you, and your classmate says nobody [everybody]

76. After considering sharing the extra tips with you, your manager at the restaurant decides to keep nothing [everything]

77. You hope not to be voted out of the group and you aren't [are]

78. You ask the professor how many students are doing better than you, and the professor says none [all]

79. You ask what you should change about your essay, and the TA says to change nothing [everything]

80. You ask your friend if your clothes look that bad and your friend says no [yes]

81. You ask your roommate not to lend anyone your DVDs, and your roommate doesn't [does]

82. You ask your classmate not to copy off your exam and your roommate doesn't [does]

83. You ask your best friend not to date your ex, and your best friend doesn't [does]

84. You ask a friend to wait for you, and your friend does [doesn't]

85. You ask your friend not to tell anyone that you failed the exam, and your friend doesn't [does]

86. Your old friends from home say that getting along without you will be really difficult [easy]

87. Someone steals your ipod, and a classmate says that is awful [funny]

88. You do something you're ashamed of, and your closest friends say it will be something they'll try to forget [remember]

89. You get the lowest grade in the class, and your friend says that is unfair [fair]

90. A stranger knocks you over and then tries to make sure that you recover [apologize]

91. A classmate starts an argument with you and then asks for your forgiveness [apology]

92. You don't get into your top choice for graduate school, and your classmates seem upset

[happy]

93. Someone badmouths you when you aren't there, and your best friend strongly disagrees [agrees]

94. You do a comedy skit for the talent show, and your classmates say it is really funny [boring]

95. Someone posts nasty rumors about you, and your friend says they're boring [interesting]

96. If you make one small mistake, it will be ignored [remembered]

97. When you're not picked for a club, your friend says that club is the worst [best]

98. The partygoers don't want you to leave [stay]

99. You are told that admitting you to the club will be easy [difficult]

100. A co-worker tells the boss that working with you is easy [difficult]

101. You're exercising a lot and think you're in much better shape, and your friends strongly agree [disagree]

102. You ask a classmate to help you study, and your classmate says yes [no]

103. If you owe a friend a very small amount of money, your friend will probably forget

[remember] 
104. You ask a friend to bring you lunch, and your friend does [doesn't]

105. A classmate offers to save you a place in line, and he does [doesn't]

106. After promising to help you move, your friend does [doesn't] 